\title{
Características Antropométricas, Composición Corporal y Somatotipo en Tenistas ITF Elite Juniors Sudamericanos
}

\author{
Anthropometric Characteristics, Body Composition and Somatotype \\ in Elite Junior ITF Tennis Players from South America
}

Rodrigo Yáñez-Sepúlveda ${ }^{1-2}$; Samuel Díaz-Barrientos ${ }^{1}$; Sergio Montiel-González ${ }^{1}$ \& Juan Pablo Zavala-Crichton ${ }^{1}$

YÁÑEZ-SEPÚlVEDA, R.; DÍAZ-BARRIENTOS, S.; MONTIEL-GONZÁLEZ, S. \& ZAVALA-CRICHTON, J. P. Características antropométricas, composición corporal y somatotipo en tenistas ITF elite juniors sudamericanos. Int. J. Morphol., 36(3):1095-1100, 2018.

RESUMEN: El objetivo de este estudio fue determinar las características antropométricas, composición corporal y somatotipo en tenistas varones elite juniors sudamericanos de la Federación Internacional de Tenis. Participaron 25 jugadores de 17,04 \pm 1,05 años. La composición corporal se evaluó con el perfil restringido de 25 variables propuesto por Kerr en base al método pentacompartimental, el cual fracciona el cuerpo en 5 componentes (tejidos: muscular, adiposo, óseo, residual, y piel). El somatotipo se identificó con el método de Heath y Carter. Para la evaluación se utilizaron las normas propuestas por La Sociedad Internacional para el Avance de la Cineantropometría (ISAK) y el Grupo Español de Cineantropometría (GREC). El porcentaje de tejido muscular fue de 47,65 $\pm 2,83 \%$, tejido adiposo de $23,23 \pm 3,56 \%$, y tejido óseo de 11,79 \pm 1,56 \% con un índice músculoóseo(IMO) de 4,09 \pm 0,50. En cuanto al somatotipo se apreció una clasificación de tipo mesomorfo balanceado con valores de 2,5 $\pm 0,81$ de endomorfia, 4,9 $\pm 0,66$ de mesomorfia, y 2,7 \pm 0,96 de ectomorfia. El grupo de tenistas presentó un predominio del componente mesomorfia con un equilibrio entre los componentes endomorfia y ectomorfia con características de composición corporal similares a las encontradas en la literatura. El presente estudio entrega datos referenciales sobre las características antropométricas en tenistas sudamericanos nivel elite categoría junior.

PALABRAS CLAVE: Composición corporal; Somatotipo; Tenis; ITF Junior.

\section{INTRODUCCIÓN}

El Tenis es un deporte profesional olímpico muy popular que cuenta con 210 países miembros afiliados a la Federación Internacional de Tenis (International Tennis Federation, 2018). Se caracteriza por una elevada exigencia perceptivo-motriz y alta velocidad de ejecución y precisión, en el cual el deportista debe tomar decisiones rápidas y ser preciso para reducir las imprecisiones en el juego (Lara Bocanegra, 2014; Hoppe et al., 2016). Las personas que practican disciplinas de raqueta necesitan mejorar constantemente sus cualidades físicas con el fin de competir al máximo nivel, por lo tanto, es necesario conseguir y mantener en dichos jugadores una óptima composición corporal, no obstante, existen pocos estudios publicados relacionados con esta temática (Martínez-Rodríguez et al., 2015). En el deporte de elite durante los últimos años, las características antropométricas y la composición corporal asociadas al ren- dimiento deportivo han tomado una gran importancia (Henríquez-Olguín et al., 2013; Gale-Watts \& Nevill, 2016). Diversas investigaciones han estudiado los perfiles antropométricos de distintos deportes, los cuales han logrado establecer características específicas que sirven como datos referenciales, permitiendo identificar las características de los deportistas en distintos niveles de rendimiento (Rienzi et al., 2000; Sánchez-Muñoz et al., 2007; Holway \& Garavaglia, 2009; Chaabène et al., 2012; Vernillo et al., 2013; Erculj \& Bracic, 2014; Rodriguez et al., 2014; Gutnik et al., 2015; Yáñez Sepúlveda et al., 2015; Bridge et al., 2016; Shovlin et al., 2018), en este escenario el estudio del somatotipo y la composición corporal cobra importancia, ya que cada especialidad deportiva presenta una serie de exigencias que obliga, en la mayoría de los casos, a poseer una determinada anatomía en los deportistas con el fin de

${ }^{1}$ Pedagogía en Educación Física, Universidad Andrés Bello, Chile. Calle Quillota $N^{\circ} 980$ Viña del Mar, Chile.

${ }^{2}$ Facultad de Filosofía y Educación, Pontificia Universidad Católica de Valparaíso, Chile. 
lograr un desempeño deportivo óptimo (Rodríguez et al., 2014). Por ejemplo, en lo que respecta a las características específicas en el desarrollo del tenista, la altura es el determinante antropométrico principal de la velocidad de saque en jugadores profesionales (Bonato et al., 2015). En lo que concierne a la valoración antropométrica, en la actualidad existen diversos métodos para evaluar la composición corporal, sin embargo, no todos están al alcance de los deportistas. Uno de los métodos más utilizados y de fácil acceso es la antropometría, la cual permite obtener datos relevantes sobre la composición corporal, los que pueden ser reproducidos y comparados con otros atletas de la disciplina deportiva (Martínez-Rodríguez et al.), en este sentido, la técnica de evaluación del somatotipo permite estimar la forma corporal y su distribución, brindando un resumen cuantitativo del físico, como un total unificado (Norton \& Olds, 1996). En el Tenis la investigación actual no ha dejado claro los parámetros de referencia actuales en cuanto al somatotipo y composición corporal. En base a lo señalado el objetivo del presente estudio fue determinar las características antropométricas, composición corporal y el somatotipo en 25 tenistas International Tennis Federation (ITF) elite juniors sudamericanos con la finalidad de obtener evidencia referencial para la toma de decisiones.

\section{MATERIAL Y MÉTODO}

Diseño y participantes. Investigación descriptiva que consideró una muestra de 25 jugadores de Tenis categoría juniors elite sudamericanos pertenecientes al ranking de la Federación Internacional de Tenis (ITF), la edad promedio de la muestra fue de 17,04 $\pm 1,05$ años y de género masculino. Dichos jugadores participaron de manera voluntaria en el estudio, con la previa autorización de consentimiento informado. Las evaluaciones fueron realizadas durante el Torneo internacional de Tenis ITF Juniors y COSAT realizado en Viña del Mar durante el año 2017.

Antropometría. Se evaluó un total de 25 variables antropométricas por cada sujeto, entre ellas se encontraban las variables básicas (3): peso corporal (kg), talla sentado, y talla de pie (cm); diámetros óseos (6): biacromial, tórax transverso, tórax anteroposterior, biiliocrestídeo, humeral y femoral; perímetros corporales (10): cabeza, brazo relajado, brazo flexionado en tensión, antebrazo, tórax mesoesternal, cintura, cadera, muslo máximo, muslo medio, pantorrilla y pliegues cutáneos (6): tríceps, subescapular, supraespinal, abdominal, muslo frontal y pierna medial; Para obtener el registro de las medidas anteriormente señaladas, se utilizaron los protocolos pro- puestos por la Sociedad Internacional para el Avance de la Cineantropometría (ISAK) (Marfell-Jones et al., 2006) y el Grupo Español de Cineantropometría (GREC). Respecto a los instrumentos utilizados durante las evaluaciones, se utilizó para medir el peso corporal una balanza digital TANITA® (Japón) con precisión de 100 gramos. La talla corporal sentado y de pie, fue medida con un estadiómetro portátil CESCORF® (Sao Paulo, Brasil), donde los evaluados debían estar descalzos, además para la talla sentado se ocupó un cajón antropométrico de 50 centímetros de alto. Para el marcaje se utilizó un lápiz dermográfico de color negro COMED ${ }^{\circledR}$ (Estrasburgo, Francia). Los diámetros óseos fueron medidos con un antropómetro corto y uno largo del kit de antropometría Health \& Performance ${ }^{\circledR}$, en la medición de los perímetros se utilizó una cinta métrica metálica marca LUFKIN® (Ohio, Estados Unidos) con precisión de 1 milímetro.y por último en la medición de los pliegues cutáneos se utilizó un plicómetro marca SLIM GUIDE® (Estados Unidos) con precisión de 1 milímetro. En cuanto a la determinación de los 5 componentes del fraccionamiento de la masa corporal (tejidos:muscular,adiposo,óseo,residual y piel), se utilizó el método propuesto por Kerr y Ross (Kerr, 1988; Ross \& Kerr, 1991); cuyos resultados fueron interpretados por medio de una planilla del programa Microsoft office Excel.El índice músculo/óseo se calculo dividiendo el tejido muscular con el tejido óseo en kilogramos (músculo/ óseo). El análisis y distribución del somatotipo se efectuó a través del método propuesto por Heath y Carter (Carter \& Heath; Carter, 2002). Por último los Z score del Phantom se establecieron a través de las fórmulas de Ross \& Wilson (1974).

Confidencialidad y consideraciones éticas. Todos los procedimientos realizados durante la investigación consideraron las recomendaciones éticas de la declaración de Helsinki para investigación en seres humanos (World Medical Asociation, 2013). Las evaluaciones se realizaron previo consentimiento informado autorizado por los entrenadores o apoderados de los deportistas donde se presentaron los objetivos, metodología y alcances del estudio.

Análisis estadístico. Para la descripción de las variables se utilizaron los estadísticos media y desviación estándar, los que fueron tabulados y calculados con los programas Microsoft Office Excel versión 2013® (Albuquerque, Estados Unidos) y Graphpad Prism versión 7.0®. (California, Estados Unidos) respectivamente, el somatotipo fue calculado con el software Somatotype Calculation and Analysis Software versión 1.1 (San Diego, CA, EE.UU). El análisis de fiabilidad se realizó con el software SPSS versión 23.0 para Windows®. 


\section{RESULTADOS}

Participaron 25 tenistas juniors de nivel sudamericano elite con una edad de 17,04 $\pm 1,05$ años, representantes de países como Argentina, Chile, Brasil, Ecuador, Perú y Uruguay. Para validar el método se realizó un análisis de fiabilidad considerando el coeficiente de correlación intraclase (CCI) que fue de 0,98 (IC $95 \%$; 0,95- 0,99) $(\mathrm{p}=0,000)$, un nivel de correlación de Pearson(r) de 0,96 $(\mathrm{p}=0,000)$ y un coeficiente de determinación (R2) de 0,92 en la comparación de las variables peso corporal estimado y peso corporal bruto en referencia a método pentacompartimental (Kerr). Los resultados muestran que el método presenta concordancia y correlación para ser utilizado en la muestra. Considerando la distribución de todos los componentes estudiados y representados en las siguientes tablas y figuras se representan los valores de los resulta- dos obtenidos en cada variable, finalmente se muestra la distribución en la somatocarta con el resultado promedio de los deportistas que participaron del estudio.

En la tabla I se observan los resultados de los valores medios y desviación estándar sobre las variables básicas (talla, peso, edad, IMC), $\sum$ de seis pliegues, índice músculo/óseo pliegues, diámetros y perímetros obtenidos en las evaluaciones antropométricas en los tenistas sudamericanos juniors elite.

En la Tabla II se presentan los resultados de los valores medios y desviación estándar de los 5 componentes del método pentacompartimental, y el somatotipo de los 25 tenistas evaluados; los cuales están representados en porcentajes y kilogramos.

Tabla I. Variables básicas, pliegues, diámetros y perímetros en el grupo de tenistas evaluados.

\begin{tabular}{|c|c|c|}
\hline \multicolumn{2}{|r|}{ Variable } & Tenista Junior elite sudamericanos \\
\hline \multirow{7}{*}{ Variables básicas } & Edad (años) & $17,04 \pm 1,05$ \\
\hline & Talla (cm) & $176,08 \pm 6,30$ \\
\hline & Talla sentado $(\mathrm{cm})$ & $91,53 \pm 3,27$ \\
\hline & Peso corporal (kg) & $70,13 \pm 7,70$ \\
\hline & Peso estructurado & $70,51 \pm 9,61$ \\
\hline & IMC (Peso/Estatura_) & $23,06 \pm 2,40$ \\
\hline & _ de 6 pliegues $(\mathrm{mm})$ & $55,34 \pm 17,84$ \\
\hline \multirow{8}{*}{ Pliegues } & Índice músculo/óseo & $4,09 \pm 0,50$ \\
\hline & Tríceps & $9,42 \pm 3,02$ \\
\hline & Subescapular & $8,08 \pm 1,66$ \\
\hline & Supraespinal & $8,46 \pm 3,63$ \\
\hline & Abdominal & $11,76 \pm 5,66$ \\
\hline & Muslo frontal & $10,6 \pm 3,50$ \\
\hline & Pantorrilla & $7,02 \pm 2,53$ \\
\hline & Biacromial & $39,90 \pm 1,87$ \\
\hline \multirow{4}{*}{ Diámetros } & Tórax transverso & $28,65 \pm 2,13$ \\
\hline & Tórax antero-posterior & $18,54 \pm 1,43$ \\
\hline & Biiliocrestideo & $27,17 \pm 1,72$ \\
\hline & Humeral & $7,20 \pm 0,32$ \\
\hline \multirow{11}{*}{ P erímetros } & Femoral & $9,71 \pm 0,46$ \\
\hline & Cabeza & $55,72 \pm 1,67$ \\
\hline & Brazo relajado & $28,89 \pm 2,07$ \\
\hline & Brazo flexionado en tensión & $31,30 \pm 2,06$ \\
\hline & Antebrazo & $27,32 \pm 1,44$ \\
\hline & Tórax mesoesternal & $92,26 \pm 4,38$ \\
\hline & Cintura & $79,33 \pm 4,50$ \\
\hline & Cadera & $93,23 \pm 4,29$ \\
\hline & Muslo medio & $52,24 \pm 2,87$ \\
\hline & Pantorrilla & $36,92 \pm 1,66$ \\
\hline & Muslo máximo & $56,6 \pm 3,31$ \\
\hline
\end{tabular}


Tabla II. Tejidos adiposo, muscular, residual, óseo, piel y componentes endomorfia, mesomorfia y ectomorfia en el grupo de tenistas evaluados.

\begin{tabular}{lcc}
\hline Variable & \multicolumn{2}{c}{ Tenistas Junior elite sudamericanos } \\
\hline & $\%$ & $23,23 \pm 3,56$ \\
Tejido muscular & $(\mathrm{Kg})$ & $16,30 \pm 3,20$ \\
& $\%$ & $47,65 \pm 2,83$ \\
Tejido residual & $(\mathrm{Kg})$ & $33,44 \pm 4,16$ \\
& $\%$ & $11,91 \pm 1.02$ \\
Tejido óseo & $(\mathrm{Kg})$ & $8,36 \pm 1,21$ \\
& $\%$ & $11,79 \pm 1,56$ \\
Tejido piel & $(\mathrm{Kg})$ & $8,26 \pm 1,31$ \\
& $(\mathrm{Kg})$ & $5,39 \pm 0,38$ \\
Somatotipo & Endomorfia & $3,76 \pm 0,29$ \\
& Mesomorfia & $2,5 \pm 0,81$ \\
& Ectomorfia & $4,99 \pm 0,66$ \\
& & $2,74 \pm 0,96$ \\
\hline
\end{tabular}

En la Figura 1 se aprecia la somatocarta y distribución del somatotipo de los 25 tenistas sudamericanos junior. Se presenta el somatotipo medio, el que se clasificó como mesomorfo balanceado 2,5-4,9-2,7.

\section{DISCUSIÓN}

Los resultados de esta investigación muestran un somatotipo promedio con una clasificación mesomorfo balanceado 2,5-4,9-2,7, en cuanto a la composición corporal, el tejido muscular fue de 47,65 $\pm 2,83 \%(33,44 \pm 4,16 \mathrm{~kg})$ y el tejido adiposo de 23,23 $\pm 3,56 \%(16,30 \pm 3,20 \mathrm{~kg})$. La revisión de la literatura muestra que son pocos los estudios en esta población, en este contexto, un estudio realizado en 123 jugadores de élite junior ( 57 hombres y 66 mujeres), el somatotipo medio de jugadores masculinos se definió también como mesomorfo balanceado $(2,4-5,2-2,9)$, el porcentaje del tejido muscular fue de 45,2 $\pm 1,5 \%$ y en el tejido adiposo de 28,5 $\pm 3,7 \%$ (Sánchez-Muñoz et al.), presentando por parte de estos un menor porcentaje de tejido muscular, y mayor porcentaje de tejido adiposo en relación a los resultados del presente estudio. Otro estudio donde participaron 550 tenistas de diferentes categorías, edad y sexo; de los cuales 240 participantes evaluados pertenecían a la población masculina, los resultados mostraron un somatotipo que se distribuyó como mesomorfo-ectomorfo 2,4-4,1-3,7 (Solanellas et al., 1996). En relación a otros deportes de raqueta, en un estudio realizado en jugadores Españoles de Bádminton, en el cual participaron 31 hombres $(21,7 \pm 4,3$ años) y 15 mujeres (19,1 $\pm 4,4$ años), los resultados evidenciaron que el somatotipo en varones pertenecía a la clasificación de ecto-mesomorfo 2,2-3,7--2,8, y en las mujeres

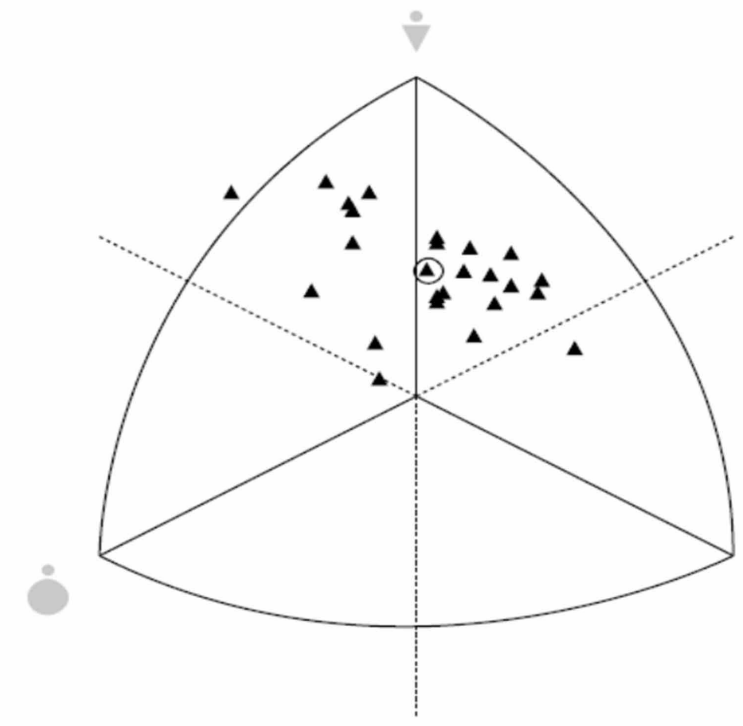

$\mathbf{\Delta}=$ Dispersión por deportista ; $\bigcirc$ = Promedio del grupo.

Fig. 1. Somatocarta de los tenistas sudamericanos ITF junior.

una clasificación de mesomorfo-endomorfo 3,4-- 3,6 --2,1. En cuanto a la composición corporal, los hombres presentaron $50,86 \%$ de tejido muscular y $8,35 \%$ de tejido adiposo (Abián et al., 2012), siendo mayor el porcentaje de tejido muscular y menor el porcentaje de tejido adiposo en comparación a los resultados obtenidos. Por otra parte, una investigación que comparó el somatotipo de 21 jugadores varones categoría adulto de Pádel y 26 jugadores de Tenis, no se encontraron diferencias significativas; presentando ambos un somatotipo endo-mesomorfo. En la composición corporal, los jugadores de Tenis presentaron 33,2 $\pm 3,6(\mathrm{Kg})$ de masa muscular y 16,2 \% de tejido adiposo; por otro lado, los jugadores de Pádel 32,4 \pm 3,0 (Kg) de tejido muscular y $18,3 \%$ de tejido adiposo (Martínez-Rodríguez et al.). Un estudio realizado en Chile, en el cual se evaluó una muestra de 50 jugadores de tenis de mesa de nivel competitivo, con un promedio de edad 21,6 $\pm 3,1$ años, se encontró en los jugadores una clasificación del somatotipo con características endo-mesomorfo (4,1-4,9-1,8) (Yáñez Sepúlveda et al.). Así mismo, en otro estudio realizado en 31 jugadores internacionales de Tenis de mesa de alto nivel (18 varones y 13 mujeres), los hombres presentaron una clasificación del somatotipo mesomorfo balanceado (2,5-4,6-2,6), al igual que la clasificación encontrada en este trabajo. Respecto a la composición corporal, al comparar los individuos de sexo masculino con el femenino se encontraron diferencias significativas en el porcentaje de peso graso y muscular; presentando en este último por parte de ambos grupos valores 
por encima del $45 \%$, siendo ligeramente superior en varones $46,9 \%$ frente a las mujeres $45,7 \%$ (Pradas de la Fuente et al., 2013). Una investigación que describió el somatotipo de 48 jugadores de alto rendimiento de Pádel (36 hombres y 12 mujeres) evidenció un somatotipo promedio con clasificación endo-mesomorfo en hombres $(3,6-6,9-1,9)$ (Castillo-Rodríguez et al., 2014). Por último, según un estudio donde se relacionaron las variables fisiológicas que intervenían en los jugadores de squash durante una competición, se evaluaron 13 jugadores de este deporte; en los cuales los componentes del somatotipo medio que presentaron tuvo una distribución de endo-mesomorfo (3,5-5,7-1,2) (Alvero et al., 2006), resultados que discrepan con la clasificación obtenida en este estudio. En lo que respecta al IMC, se ha convertido en un factor importante asociado al éxito cuando este refleja una mayor masa muscular en lugar de una mayor adiposidad, identificado por un término de interacción significativamente positivo (más pronunciado) en jugadores de Tenis que consiguen mejores resultados por año. Es por esto que en los últimos años la masa muscular se ha transformado en un factor importante asociado con el éxito en todos los torneos de Grand Slam (Gale-Watts \& Nevill), esto fundamenta la búsqueda de un somatotipo en el Tenis orientado a un mayor desarrollo (no excesivo) del tejido muscular en sujetos que alcanzan elevadas estaturas.

YÁÑEZ-SEPÚLVEDA，R.; DÍAZ-BARRIENTOS，S.; MONTIEL-GONZÁLEZ, S. \& ZAVALA-CRICHTON, J. P. Anthropometric characteristics, body composition and somatotype in elite junior ITF tennis players from South America. Int. J. Morphol., 36(4):1095-1100, 2018.

SUMMARY: The objective of the study was to determine the anthropometric characteristics, body composition and somatotype in male elite junior tennis players from the International Tennis Federation (ITF). Twenty five players of $17.04 \pm 1.05$ years participated. The body composition was evaluated with the restricted profile of 25 variables proposed by Kerr based on the pent compartmental method, which divided the body into 5 components (tissues: muscle, adipose, bone, residual, and skin). The somatotype was identified with the Heath and Carter method. For the evaluation, the norms proposed by the International Society for the Advancement of Cineanthropometry (ISAK) and the Spanish Group of Cineanthropometry (GREC) were used. The percentage of muscle tissue was $47.65 \pm 2.83 \%$, adipose tissue of $23.23 \pm$ $3.56 \%$, and bone tissue of $11.79 \pm 1.56 \%$ with a muscle-osseous index (IMO) of $4.09 \pm 0.50$. Regarding somatotype, a balanced mesomorphic classification was observed with values of $2.5 \pm 0.81$ of endomorphy, $4.9 \pm 0.66$ of mesomorphy, and $2.7 \pm 0.96$ of ectomorphy. The group of tennis players presented a predominance of the mesomorphy component with a balance between the components endomorphy and ectomorphy with characteristics of body composition similar to those found in the literature. The present study provides referential data on the anthropometric characteristics in South American tennis players elite junior category.

KEY WORDS: Body composition; Somatotype; TennisITF Junior.

\section{REFERENCIAS BIBLIOGRÁFICAS}

Abián, P.; Abián-Vicén, J. \& Sampedro J. Anthropometric analysis of body symmetry in badminton players. Int. J. Morphol., 30(3):945-51, 2012.

Alvero, J.; Barrera, J. \& Mesa, A. Correlations of physiological responses in squash players during competition. En: Lees, A.; Cabello, D. \& Torres, G. (Eds). Science and Racket Sports IV. Madrid, 2006. pp.64-9.

Bonato, M.; Maggioni, M. A.; Rossi, C.; Rampichini, S.; La Torre, A. \& Merati, G. Relationship between anthropometric or functional characteristics and maximal serve velocity in professional tennis players. J. Sports Med. Phys. Fitness., 55(10):1157-65, 2015.

Bridge, C. A.; Ferreira da Silva Santos, J.; Chaabène, H.; Pieter, W. \& Franchini, E. Physical and physiological profiles of taekwondo athletes. Sports Med., 44(6):713-33, 2016.

Carter, J. E. L. \& Heath, B. H. Somatotyping-Development and Applications. Cambridge, Cambridge University Press, 1990. pp.398-420.

Carter, J. E. L. The Heath-Carter Anthropometric Somatotype. Instruction Manual. San Diego, Department of Exercise and Nutritional Sciences, San Diego State University, 2002.

Castillo-Rodríguez, A.; Hernández-Mendo, A. \& Alvero-Cruz, J. R. Morphology of the elite paddle player - Comparison with other racket sports. Int. J. Morphol., 32(1):177-82, 2014.

Chaabène, H.; Hachana, Y.; Franchini, E.; Mkaouer, B. \& Chamari, K. Physical and physiological profile of elite karate athletes. Sports Med., 42(10):829-43, 2012.

Erculj, F. \& Bracic, M. Morphological profile of different types of top young female European basketball players. Coll. Antropol., 38(2):51723, 2014.

Gale-Watts, A. S. \& Nevill, A. M. From endurance to power athletes: The changing shape of successful male professional tennis players. Eur. $J$. Sport Sci., 16(8):948-54, 2016.

Gutnik, B.; Zuoza, A.; Zuoziene ', I.; Alekrinskis, A.; Nash, D. \& Scherbina, S. Body physique and dominant somatotype in elite and low-profile athletes with different specializations. Medicina (Kaunas), 51(4):247$52,2015$.

Henríquez-Olguín, C.; Báez, E.; Ramírez-Campillo, R. \& Cañas, R. Somatotype profile of professional male soccer chilean players. Int. J. Morphol., 31(1):225-30, 2013.

Holway, F. E. \& Garavaglia, R. Kinanthropometry of Group I rugby players in Buenos Aires, Argentina. J. Sports Sci., 27(11):1211-20, 2009.

Hoppe, M. W.; Baumgart, C.; Hilberg, T.; Freiwald, J. \& Wehmeier, U. F. Changes of standard physiological-perceptual markers and circulating MicroRNAs in response to tennis match-play: A case report of two elite players. J. Hum. Kinet., 51:71-81, 2016.

International Tennis Federation (ITF). Membership Status. Londres, International Tennis Federation (ITF), 2018. Disponible en: http:// www.itftennis.com/media/276275/276275.pdf

Kerr, D. An Anthropometric Method for Fractionation of Skin, Adipose, Bone, Muscle and Residual Masses in Males and Females Age 6 to 77 Years. M. Sc. Kinesiology Tesis. British Columbia, Simon Fraser University, 1988.

Lara Bocanegra, A. Tenis. Ejercicios progresivos para desarrollar tu juego. G. Torres Luque, A. Sánchez Pay, C. Gago Fuentes y J. Ros García (2013). Badalona: Paidotribo. Cuad. Psicol. Deporte, 14(2):155-156, 2014. 
YÁÑEZ-SEPÚLVEDA, R.; DÍAZ-BARRIENTOS, S.; MONTIEL-GONZÁLEZ, S. \& ZAVALA-CRICHTON, J. P. Características antropométricas, composición corporal y somatotipo en tenistas ITF elite juniors sudamericanos. Int. J. Morphol., 36(3):1095-1100, 2018

Marfell-Jones, M.; Olds, T.; Stewart, A. \& Carter, L. International Standards for Anthropometric Assessment. Potchefstroom, International Society for the Advancement of Kinanthropometry, 2006.

Martínez-Rodríguez, A.; Roche Collado, E. \& Vicente-Salar, N. Body composition assessment of paddle and tennis adult male players. Nutr. Hosp., 31(3):1294-301, 2015.

Norton, K. \& Olds, T. Antropométrica. Sydney, University of New Sawth Wales Press, 1996.

Pradas de la Fuente, F.; González Jurado, J. A.; Molina Sotomayor, E. \& Castellar Otín, C. Anthropometric characteristics, body composition and somatotype of high level table tennis players. Int. J. Morphol., 31(4):1355-64, 2013.

Rienzi, E.; Drust, B.; Reilly, T.; Carter, J. E. \& Martin, A. Investigation of anthropometric and work-rate profiles of elite South American international soccer players. J. Sports Med. Phys. Fitness, 40(2):1629, 2000.

Rodríguez, P. X.; Castillo, V. O.; Tejo, C. J. \& Rozowski, N. J. Somatotipo de los deportistas de alto rendimiento de Santiago, Chile. Rev. Chil. Nutr., 41(1):29-39, 2014

Ross, W. D. \& Kerr, D. A. Fraccionamiento de la masa corporal: Un nuevo método para utilizar en nutrición clínica y medicina deportiva. Apunt. Med. Esport, 28(109):175-88, 1991.

Ross, W. D. \& Wilson, N. C. A strategem for proportional growth assessment. Acta Paediatr. Belg., 28 suppl.:169-82, 1974.

Sánchez-Muñoz, C.; Sanz, D. \& Zabala, M. Anthropometric characteristics, body composition and somatotype of elite junior tennis players. $\mathrm{Br} . \mathrm{J}$. Sports Med., 41(11):793-9, 2007.

Shovlin, A.; Roe, M.; Malone, S. \& Collins, K. Positional anthropometric and performance profile of elite gaelic football players. J. Strength Cond. Res., 32(8):2356-62, 2018.

Solanellas, F.; Tuda, M. \& Rodríguez, F. A. Valoración cineantropométrica de tenistas de diferentes categorías. Apunt. Educ. Fís. Deportes, (4445):122-35, 1996.

Vernillo, G.; Schena, F.; Berardelli, C.; Rosa, G.; Galvani, C.; Maggioni, M.; Agnello, L. \& La Torre, A. Anthropometric characteristics of topclass Kenyan marathon runners. J. Sports Med. Phys. Fitness, 53(4):4038,2013

World Medical Association (WMA). Declaration of Helsinki. Amended by the $64^{\text {th }}$ WMA General Assembly, Fortaleza, WMA Archives, FerneyVoltaire, 2013.

Yáñez Sepúlveda, R.; Barraza, F.; Rosales Soto, G.; Báez, E. \& Tuesta, M. Anthropometric chilean table tennis players of competitive features. Nutr. Hosp., 32(4):1689-94, 2015.

\section{Dirección para correspondencia: \\ Rodrigo Yáñez-Sepúlveda \\ Pedagogía en Educación Física \\ Universidad Andrés Bello \\ Calle Quillota № 980 \\ Viña del Mar \\ CHILE}

Email: fach.efi@gmail.com

Recibido : 17-01-2018

Aceptado: 01-06-2018 